\title{
A comparison between left molar direct laryngoscopy and the use of a Bonfils intubation fibrescope for tracheal intubation in a simulated difficult airway
}

\section{Comparaison entre la laryngoscopie directe rétromolaire gauche et l'utilisation d'un fibroscope d'intubation de Bonfils pour l'intubation trachéale dans des cas simulés d'intubation difficile des voies aériennes}

\author{
Anju Gupta, MD · Seema Thukral, MD • \\ Archana Lakra, MD • Sushil Kumar, MBBS \\ Received: 7 February 2014/ Accepted: 2 February 2015/Published online: 14 February 2015 \\ (C) Canadian Anesthesiologists' Society 2015
}

\begin{abstract}
Background Tracheal intubation in patients with an immobilized cervical spine can be difficult because of a restricted mouth opening and limited neck movements. Use of the Bonfils intubation fibrescope (BIF) or left molar (LM) laryngoscopy may be suitable options for tracheal intubation in such patients. Intubation adjuncts, such as an endotracheal tube introducer, may improve the overall intubation success rate with the LM approach. Formal studies are currently lacking on the use of LM laryngoscopy with a tube introducer.
\end{abstract}

Methods After Institutional Review Board approval, a cervical collar (to simulate a difficult airway scenario) was

This report was previously presented at the International Anesthesia Research Society (IARS) 2013, San Diego, USA.

Author contributions Anju Gupta and Seema Thukral helped design the study and write the manuscript. Anju Gupta, Seema Thukral, Archana Lakra, and Sushil Kumar have seen the original study data and reviewed the analysis of the data. Anju Gupta, Sushil Kumar, and Archana Lakra helped conduct the study. Anju Gupta analyzed the data and is the author responsible for archiving the study files.

A. Gupta, MD

Delhi State Cancer Institute, Dilshad Garden, New Delhi, India

A. Gupta, MD ( $\square)$

437, Pocket A, Sarita Vihar, New Delhi 110076, India

e-mail: dranjugupta2009@rediffmail.com

S. Thukral, MD · A. Lakra, MD - S. Kumar, MBBS

ESI Hospital, Maa Anand Mayee Marg, New Delhi, India placed on 120 prospective elective surgical patients who were randomly assigned to tracheal intubation with a BIF (Group BIF, $n=60$ ) or with tube introducer-assisted LM laryngoscopy with routine optimal external laryngeal manipulation (Group LM, $n=60$ ). The groups were compared for the primary endpoint, total intubation time, as well as for time to glottic view, tube introducer insertion time, intubation success rate, number of intubation attempts, and airway complications.

Results The mean (SD) total time for intubation was longer in Group LM than in Group BIF [40.4 (14.2) sec vs 33.1 (15.4) sec, respectively; mean difference 7.3 sec; $99 \%$ confidence interval (CI) 3.2 to 14.4; $P<0.001]$ despite less mean (SD) total time required for glottic view [15.4 (10.3) sec vs 23.8 (15.7) sec, respectively; mean difference 8.3 sec; $99 \%$ CI 2.3 to 14.7; $P<0.001]$. The overall success rate was comparable between groups $(95.0 \%$ in Group BIF vs $96.6 \%$ in Group LM; P =0.64). Tracheal intubations could not be performed as per protocol in three patients in Group BIF and in two patients in Group LM and were considered as failures. No differences between the groups were found in the incidence of side effects.

Conclusion The tube introducer-assisted LM approach to intubation may be a good alternative to the BIF approach in patients with anticipated and unanticipated difficult airway scenarios.

\section{Résumé}

Contexte Chez les patients ayant une immobilisation de la colonne cervicale, l'intubation trachéale peut être difficile en raison d'une ouverture restreinte de la bouche 
et de la limitation des mouvements du cou. L'utilisation d'un fibroscope d'intubation de Bonfils (BIF) ou d'une laryngoscopie rétromolaire gauche (LM) peut être une option convenant à l'intubation trachéale chez de tels patients. Les additifs à l'intubation, tels qu'un introducteur de tube endotrachéal, peuvent améliorer le taux de succès global de l'intubation dans le cas de la technique LM. Nous manquons actuellement d'études formelles sur l'emploi de la laryngoscopie LM avec introducteur de tube.

Méthodes Après approbation du Comité d'éthique de la recherche de l'établissement, un collier cervical (visant à simuler un scénario d'intubation difficile) a été placé sur 120 patients prospectifs devant subir une chirurgie élective et répartis aléatoirement pour avoir une intubation trachéale selon la technique BIF (groupe BIF, $n=60$ ) ou la technique de laryngoscopie LM aidée par un introducteur de tube avec manipulation laryngée externe optimale (groupe LM, $n=60$ ). La comparaison entre les groupes a inclus le critère d'évaluation principal, le temps total d'intubation, ainsi que le temps d'exposition de la glotte, le temps d'insertion de l'introducteur de tube, le taux de succès de l'intubation, le nombre de tentatives d'intubation et les complications au niveau des voies aériennes.

Résultats Le temps total moyen (ET) pour l'intubation a été plus long dans le groupe LM que dans le groupe BIF (respectivement 40,4 2,14 secondes contre 33,1 4,15 secondes; différence moyenne 7,3 sec; intervalle de confiance (IC) à $99 \%: 3,2$ à 14,4; $P<0,001)$ en dépit du fait que le temps total moyen (ET) nécessaire à l'exposition de la glotte ait été moindre (respectivement, 15,4 3,10 sec contre 23,8 7,15 sec; différence moyenne 8,3 sec; IC à $99 \%$ IC 2,3 à14,7; $P<0,001]$. Le taux de succès global a été comparable entre les groupes $(95,0 \%$ dans le groupe BIF contre 96,6 \% dans le groupe LM; $P<0,64)$. Une intubation trachéale n'a pas pu être réalisée selon le protocole chez trois patients du groupe BIF et deux patients du groupe LM; ces cas ont été assimilés à des échecs. Aucune différence n'a été constatée entre les groupes quant à l'incidence des effets indésirables.

Conclusion L'approche LM aidée par un introducteur de tube peut être une bonne solution de remplacement à la technique BIF chez les patients chez lesquels on peut s'attendre, ou non, à des difficultés d'intubation de la voie aérienne.

Direct laryngoscopy requires movement at the cervical spine to align the three axes (oral, pharyngeal, and laryngeal) for direct vision of the vocal cords. ${ }^{1}$ Application of a cervical collar reduces neck movements and mouth opening, lifts the chin, and moves the larynx anteriorly. ${ }^{1,2}$ This creates extremely challenging intubation conditions even for the experienced anesthesiologist.

The Bonfils intubation fibrescope (BIF) is a rigid reusable optical stylet that has been shown to facilitate endotracheal intubation in anticipated and unanticipated difficult airways with very good success rates. ${ }^{3-5}$ The Bonfils intubation fibrescope has a narrow shaft and a distal $40^{\circ}$ angle curve which makes it a suitable device for tracheal intubation in patients with an unstable or fixed cervical spine. ${ }^{6,7}$ In patients with a simulated difficult airway using a cervical collar, the BIF approach has shown a marked improvement in the intubation success rate when compared with the midline approach to laryngoscopy and intubation. ${ }^{4,8}$ Nevertheless, the BIF is expensive, has a shallow learning curve, and is not widely available. ${ }^{9}$

In the era of technology, a plethora of advanced airway devices are available for managing the difficult airway, ${ }^{10}$ but laryngoscopy still remains the least expensive, most readily available, and most familiar equipment to secure the airway. Nevertheless, even under optimal conditions, midline laryngoscopy may be difficult in patients with restricted neck movement and mouth opening. The molar approach to laryngoscopy reduces the distance from the patient's teeth to the larynx, minimizes intrusion of maxillary structures into the line of view, and changes the line of vision from an oblique superoinferior direction to a horizontal lateromedial direction. The glottic view is improved using a left molar (LM) approach; ${ }^{11,12}$ however, it may be difficult to intubate with the tongue bulging on the medial side. ${ }^{13}$ Intubation aids such as endotracheal tube introducers (long and thin with a curved tip) can be manipulated through the narrow space created during LM laryngoscopy and thus may improve the overall intubation success rate. Formal studies are lacking on intubation using the tube introducer-assisted LM laryngoscopy approach and on comparisons with the BIF approach to intubation. Consequently, we hypothesized that tube introducerassisted LM laryngoscopy is non inferior to the BIF approach to tracheal intubation in patients wearing a rigid cervical collar to simulate a difficult airway.

\section{Methods}

With Institutional Ethics Committee approval (ESI Hospital Ethics Committee No: 114-A-19/11/90/2011Okh on November 6, 2012; Dr. V. K. Aggarwal, Chair) and written informed consent, 120 adult patients (American Society of Anesthesiologists physical status I-II, aged 18$60 \mathrm{yr}$ ) scheduled to undergo elective surgical procedures under general anesthesia were prospectively included in the study. The exclusion criteria included all patients with anticipated difficult ventilation (presence of beard, obese 
with body mass index $>35 \mathrm{~kg} \cdot \mathrm{m}^{-2}$ snoring, edentulous, intraoral tumours, receding chin, etc.) or anticipated difficult tracheal intubation (modified Mallampati class 3-4 view, ${ }^{14}$ mouth opening $<3 \mathrm{~cm}$, thyromental distance $<6.5 \mathrm{~cm}$, and sternomental distance $<12.5 \mathrm{~cm}$ ), patients with loose molar teeth, increased risk of aspiration, pregnant patients, and those with a history of difficult intubation.

Patients were monitored with electrocardiography, noninvasive blood pressure, and pulse oximetry. Glycopyrrolate $0.2 \mathrm{mg} i v$, midazolam $0.05 \mathrm{mg} \cdot \mathrm{kg}^{-1} i v$, and fentanyl $2 \mathrm{ug} \cdot \mathrm{kg}^{-1} i v$ were given to all patients ten minutes before the procedure. After preoxygenation for three minutes, anesthesia was induced with an injection of propofol $2 \mathrm{mg} \cdot \mathrm{kg}^{-1}$; ability to perform bag and mask ventilation was ensured, and the neuromuscular blockade was achieved with vecuronium $0.1 \mathrm{mg} \cdot \mathrm{kg}^{-1} i v$. Bag and mask ventilation was performed for three minutes with an $\mathrm{O}_{2}$ : sevoflurane mixture to achieve a MAC of 1.3. Next, a rigid Ambu ${ }^{\circledR}$ Perfit ACE collar (Ambu Inc., Copenhagen, Denmark) for adults $(56 \times 18 \times 1.5 \mathrm{~cm}$ with 16 different settings) was applied with a finger sizing method using the horizontal sizing lines marked on the collar. Optimal external laryngeal manipulation (OELM) was applied through a hole in the anterior portion of the collar $(8.5 \mathrm{~cm}$ wide and $6.5 \mathrm{~cm}$ long in the neckless position; $8.5 \mathrm{~cm}$ wide and $10.5 \mathrm{~cm}$ long in the tall neck size). A blinded observer re-assessed the patient's mouth opening after induction of anesthesia in the supine position with the collar in situ (with maximal chin retraction). The patients were then randomly allocated to either the Bonfils intubation fibrescope, $5.0 \mathrm{~mm}$ external diameter with video monitor (Karl Storz Endoscopy, Tuttlingen, Germany), (Group BIF, $n=60$ ) or to left molar laryngoscopy (Macintosh laryngoscope size 3 ) with a $14 \mathrm{~F}$ $750 \mathrm{~mm}$ PRO-Breath Premium ${ }^{\mathrm{TM}}$ endotracheal tube introducer (Proact Medical limited, Corby, Northants, United Kingdom); (Group LM, $n=60$ ) using computergenerated codes that were maintained in sequentially numbered sealed opaque envelopes.

The primary endpoint in our study was the total time required for successful intubation (i.e., total time from passage of the device into the oral cavity beyond the teeth until first appearance of a regular capnograph waveform on a sidestream monitor). All intubations in both groups were performed by a single anesthesiologist experienced using each technique on more than 50 patients. ${ }^{15}$

In Group BIF, the operator inserted her left thumb into the patient's mouth behind the molar teeth and lifted the jaw vertically upwards. In some of the patients, a severely restricted mouth opening (after application of the collar) precluded thumb insertion and only a chin lift was applied. A lubricated BIF shaft was then preloaded with an endotracheal tube (ETT) (females: $7 \mathrm{~mm}$ internal diameter [ID], males: $8 \mathrm{~mm}$ ID) and introduced in midline under direct vision behind the uvula. The Cormack-Lehane grade (as seen on the monitor display) was recorded. ${ }^{16}$ The BIF tip was then maneuvered below the epiglottis to obtain a centralized glottic view. The operator then stabilized the scope, and an assistant threaded the ETT beyond the glottis under direct vision.

In Group LM, the Macintosh laryngoscope was introduced from the left corner of the patient's mouth between the upper and the lower left molars. The tip of the blade was directed posteromedial between the tongue and the tonsil up to the epiglottis, and then it was guided into the valeculla to visualize the glottis. Optimal external laryngeal manipulation was routinely applied in Group LM during the entire intubation attempt, and the CormackLehane grade was recorded before and after the application of OELM. ${ }^{17}$ Thereafter, a tube introducer was introduced under direct vision (Cormack-Lehane view 1,2) or blindly (Cormack-Lehane view 3,4) until the presence of a distal "hold up". In case of failure to negotiate the tube introducer towards the glottis from the left side, the tube introducer was removed and reinserted from the opposite corner of the mouth towards the glottis. ${ }^{18}$ The ETT was then railroaded over the tube introducer under laryngoscopic visualization. In case of difficulty in threading the ETT, its bevel was rotated at the level of glottis.

A maximum of two minutes/three attempts was allowed with each technique or until oxygen saturation decreased to $<92 \%$. These patients were then preoxygenated for three minutes, and a single attempt was allowed with the other technique. In case of failure with the crossover technique, the collar was immediately removed and intubation was performed using conventional midline laryngoscopy following the standard difficult airway algorithm as clinically indicated.

In addition to patient demographics (age, weight, height, sex), the following parameters were recorded (with all times were recorded using a stopwatch):

1. Total intubation time (as defined above)

2. Time to glottic view (time from beginning the insertion of the device until the optimal glottic view)

3. Tube introducer insertion time (time from glottic view until successful tube introducer placement)

4. ETT threading time (the time to thread the ETT over the tube introducer/BIF shaft into the trachea)

5. Number of attempts (process from introduction of the device into the oral cavity until removal)

6. All airway assessment parameters mentioned above

7. The mouth opening after induction of anesthesia and collar application 
8. The incidence of any airway morbidity (mucosal bleeding, blood on tube introducer/ETT, dental injury, lip injury)

9. Hemodynamic parameters (mean blood pressure and pulse rate) pre-induction, post induction, post laryngoscopy, and every minute for ten minutes

10. Postoperative sore throat (graded as mild, moderate, or severe) 18 at one, six, and $24 \mathrm{hr}$

Statistical analysis

Statistical analysis was performed using SPSS ${ }^{\circledR}$ software version 15 (SPSS Inc., Chicago, IL, USA). Due to a lack of published studies comparing the LM and BIF approaches in simulated difficult airways, we performed a pilot study of ten patients in each group. A difference of $8.3 \mathrm{sec}$ in mean (SD) total intubation time was observed between the two groups [BIF 34.8 (12.0) sec; LM 43.1 (13.6) sec]. Based on these results, we assumed that 48 patients would be required in each group for a non-inferiority margin of $\leq 15 \mathrm{sec}$ for total intubation times with a power of $90 \%$ (assuming $\alpha=0.05,1: 1$ ratio). We chose 60 cases for each group to account for protocol violation and failures.

The demographic characteristics and airway assessment parameters were compared using a Chi square test, and intubation characteristics were compared using the Student's $t$ test. The change in hemodynamic data was analyzed within each group and between groups using repeated measures analysis of variance and the Student's $t$ test, respectively. All data were reported as mean (SD) and mean difference with $99 \%$ confidence interval (CI). All reported $P$ values are two sided. The intubation times were not normally distributed; hence, the intubation characteristics are presented as original values as well as log-transformed values as mean (SD). The effect size is also reported as original and log-transformed values with 99\% CI. The final $P$ values represented in the tables and text are for the log-transformed data.

\section{Results}

The patient demographics, airway assessment parameters, and mouth opening after application of the cervical collar were comparable between groups (all $P>0.11$ ) (Table 1).

The mean difference in intubation times between the two groups was $7.3 \mathrm{sec}(99 \%$ CI 3.2 to $14.4 ; P<0.001)$. Both $99 \%$ CI limits were $<15 \mathrm{sec}$ which was our assumed non-inferiority margin (Fig. 2a, Table 2). The time to glottic view was less in Group LM than in Group BIF $(P<0.001)$, but the time for ETT threading was
Table 1 Demographic and airway assessment parameters

\begin{tabular}{lll}
\hline Parameters & Group LM $(n=60)$ & Group BIF $(n=60)$ \\
\hline Age $(\mathrm{yr})$ & $32.0(10.3)$ & $34.3(11.5)$ \\
ASA (I/II) & $34 / 26$ & $32 / 28$ \\
Sex $(\mathrm{M} / \mathrm{F})$ & $24 / 36$ & $19 / 41$ \\
Weight $(\mathrm{kg})$ & $52.2(10.1)$ & $55.2(10.7)$ \\
Height $(\mathrm{cm})$ & $155.8(7.8)$ & $157.7(7.6)$ \\
Mouth opening $(\mathrm{cm})$ & $4.5(0.7)$ & $4.4(0.5)$ \\
Mallampati grade $(1 / 2)$ & $25 / 35$ & $28 / 32$ \\
TMD (cm) & $7.5(0.7)$ & $7.3(0.8)$ \\
SMD (cm) & $17.7(1.6)$ & $17.2(1.7)$ \\
MO with collar $(\mathrm{cm})$ & $1.9(0.3)$ & $1.95(0.3)$ \\
\hline
\end{tabular}

Data are presented as mean (SD) or numbers $(n)$

ASA = American $\quad$ Society of Anesthesiologists; Group $\mathrm{BIF}=$ Bonfils intubation fibrescope; Group $\mathrm{LM}=$ left molar; $\mathrm{MO}=$ mouth $\quad$ opening; $\quad \mathrm{SMD}=$ sternomental distance; $\mathrm{TMD}=$ thyromental distance

significantly longer in Group LM than in Group BIF (Table 2, Fig. 2b) $(P<0.001)$.

Tracheal intubation was successful on first attempt in $70.0 \%$ of the patients in Group BIF and in $83.3 \%$ of the patients in Group LM $(P=0.11)$. Ninety-five percent of the patients in both groups achieved successful tracheal intubation by the second attempt. One patient in Group LM required three attempts. Overall, the intubation success rate with the two techniques was comparable between the two groups (Group LM: $96.6 \%$ vs Group BIF 95.0\%; $P=0.64$ ) (Table 3). All three tracheal intubation failures using the BIF approach were successfully intubated on the first attempt using the LM approach. Two cases in Group LM were considered a failure because of a protocol violation (the ETT was smaller than decided in the protocol) (Fig. 1). Esophageal intubation occurred in one patient in Group BIF and in none of the patients in Group LM.

In Group LM, OELM improved the Cormack-Lehane view from grade 3 to grade 2 in 11 patients (Table 3 ).

The hemodynamic response to intubation was comparable in the two groups. No patient in either group experienced hypoxia $(<92 \%)$, dental injury, or hoarseness. Five patients in Group BIF and ten in Group LM had mild sore throat $(P=0.25)$. Five patients in Group LM $(8.6 \%)$ and two patients in Group BIF (3.5\%) had mucosal bleeding $(P=0.44)$ (Fig. 3). A blood-smeared ETT/tube introducer was not found in any of the patients.

\section{Discussion}

The present study highlights that the tube introducerassisted LM approach to laryngoscopy resulted in longer 
Table 2 Intubation times

\begin{tabular}{llll}
\hline Parameter & $\begin{array}{l}\text { Group BIF }(n=58) \\
\text { Mean (SD) }\end{array}$ & $\begin{array}{l}\text { Group LM }(n=57) \\
\text { Mean (SD) }\end{array}$ & $\begin{array}{l}\text { Mean difference (LM-BIF) } \\
(99 \% \text { CI) }\end{array}$ \\
\hline Total time to intubation (sec) & $33.1(15.4)$ & $40.4(14.2)$ & $7.3(3.2$ to 14.4$)$ \\
Log X (total time to intubation) & $3.4(0.4)$ & $3.6(0.3)$ & $0.2(0.07$ to 0.4$)$ \\
Time to glottic view (sec) & $23.8(15.7)$ & $15.4(10.3)$ & $8.3(2.3 \text { to } 14.7)^{\#}$ \\
Log X (time to glottic view) & $3(0.6)$ & $3.6(0.3)$ & $0.4(0.2$ to 0.7$)$ \\
Bougie insertion time (sec) & NA & $12.1(9.2)$ & NA \\
Time to ETT insertion (sec) & $9.1(5.2)$ & $12.1(5.5)$ & $3(0.4$ to 5.5$)$ \\
Log X (time to ETT insertion) & $2(0.5)$ & $2.4(0.4)$ & $0.3(0.1$ to 0.5$)$ \\
\hline
\end{tabular}

Group BIF = Bonfils intubation fibrescope; Group LM = left molar; CI = confidence interval; ETT = endotracheal tube; LM-BIF = left molar-Bonfils intubation fibrescope; $\mathrm{NA}=$ not applicable

* Test applied after log transformation

\# Effect size (BIF-LM)

Table 3 Intubation results (CL grading, intubation attempts, and overall intubation success)

\begin{tabular}{llll}
\hline Parameters & $\begin{array}{l}\text { Group LM } \\
(n=60)\end{array}$ & $\begin{array}{l}\text { Group BIF } \\
(n=60)\end{array}$ & $P$ value \\
\hline $\begin{array}{c}\text { CL score without } \\
\text { OELM (1/2/3/4) }\end{array}$ & $0 / 48 / 12 / 0$ & $31 / 23 / 6 / 0$ & NA \\
$\begin{array}{c}\text { CL score with } \\
\text { OELM (1/2/3/4) }\end{array}$ & $2 / 57 / 1 / 0$ & NA & NA \\
$\begin{array}{c}\text { Intubation attempts } \\
(1 / 2 / 3 / \text { failure })\end{array}$ & $42 / 15 / 0 / 3$ & $50 / 7 / 1 / 2$ & 0.11 \\
$\begin{array}{c}\text { Overall intubation } \\
\text { success }\end{array}$ & $57 / 60(95.0 \%)$ & $58 / 60(96.6 \%)$ & 0.64 \\
\hline
\end{tabular}

$\mathrm{CL}=$ Cormack and Lehane; Group LM = left molar; Group BIF = Bonfils intubation fibrescope; $\mathrm{NA}=$ not applicable; $\mathrm{OELM}=$ optimal external laryngeal manipulation

intubation times than midline BIF-guided intubation despite a faster glottic view in patients with simulated difficult airway.

In patients with their cervical spine immobilized with a rigid collar, the airway management may be rendered difficult because of reduced mouth opening and limited neck movements. ${ }^{19,20}$ Both the BIF and LM approach (without adjuncts like a tube introducer) have been used independently in a simulated difficult airway with good success rates, ${ }^{4,11}$ but studies are lacking regarding their relative efficacy. The molar approach to laryngoscopy reduces the distance from the patient's teeth to the larynx, minimizes the intrusion of maxillary structures into the line of view, and changes the line of vision from an oblique superoinferior direction to a horizontal lateromedial direction. Consequently, a good glottic view can be obtained with minimal neck movement. ${ }^{11,12}$ Additionally, the BIF approach does not require excessive manipulation of the cervical spine and has been found suitable for intubation in similar cases with a simulated difficult airway. ${ }^{4}$
We had used an Ambu Perfit ACE collar with a wider chin portion, which significantly decreased the jaw excursion and mouth opening (Table 1) and created extremely challenging intubation conditions. The BIF has a narrow profile and requires a mouth opening just above the outer diameter (OD) of the ETT for intubation. In our study, we used size 7 (OD $9.6 \mathrm{~mm}$ ) and 8 (OD $11 \mathrm{~mm}$ ) ETTs for female and male patients, respectively; hence, the mean (SD) mouth opening in Group BIF [1.9 $(0.3) \mathrm{cm}]$ was sufficient for intubating with this approach. Similarly with the LM approach, since there is a short distance from the mouth to the glottic opening, we had to insert $1 / 2-2 / 3$ the length of a standard size- 3 Macintosh blade to obtain optimal glottic view. The height of the blade was approximately $1.6-1.8 \mathrm{~cm}$ at these points which was more than the mean (SD) mouth opening achieved in Group LM $[1.9(0.3) \mathrm{cm}]$.

The mean intubation times were less with the BIF approach despite a significantly faster glottic view with the LM approach $(P<0.001)$. The additional step of inserting a tube introducer in Group LM and an improved glottic view obtained in Group BIF might have contributed to faster intubation. The difference of $7.3 \mathrm{sec}$ in mean intubation times between the two groups was within our assumed non-inferiority margin. The mean intubation time with the BIF approach $(33 \mathrm{sec})$ is in agreement with the results of previous studies. ${ }^{3,5,21}$ The time to intubation with the LM approach cannot be compared with previous studies because of a paucity of previous data. In a metaanalysis, the overall rate of successful intubations using the BIF approach in patients with a difficult airway was reported to be $95.6 \%(66 / 69){ }^{22}$ In our study, BIF-guided intubation was successful in $95.0 \%$ (57/60) of patients with a simulated difficult airway using a rigid cervical collar.

Common causes of failure with the BIF approach include an inability to locate the glottic inlet, secretions, 


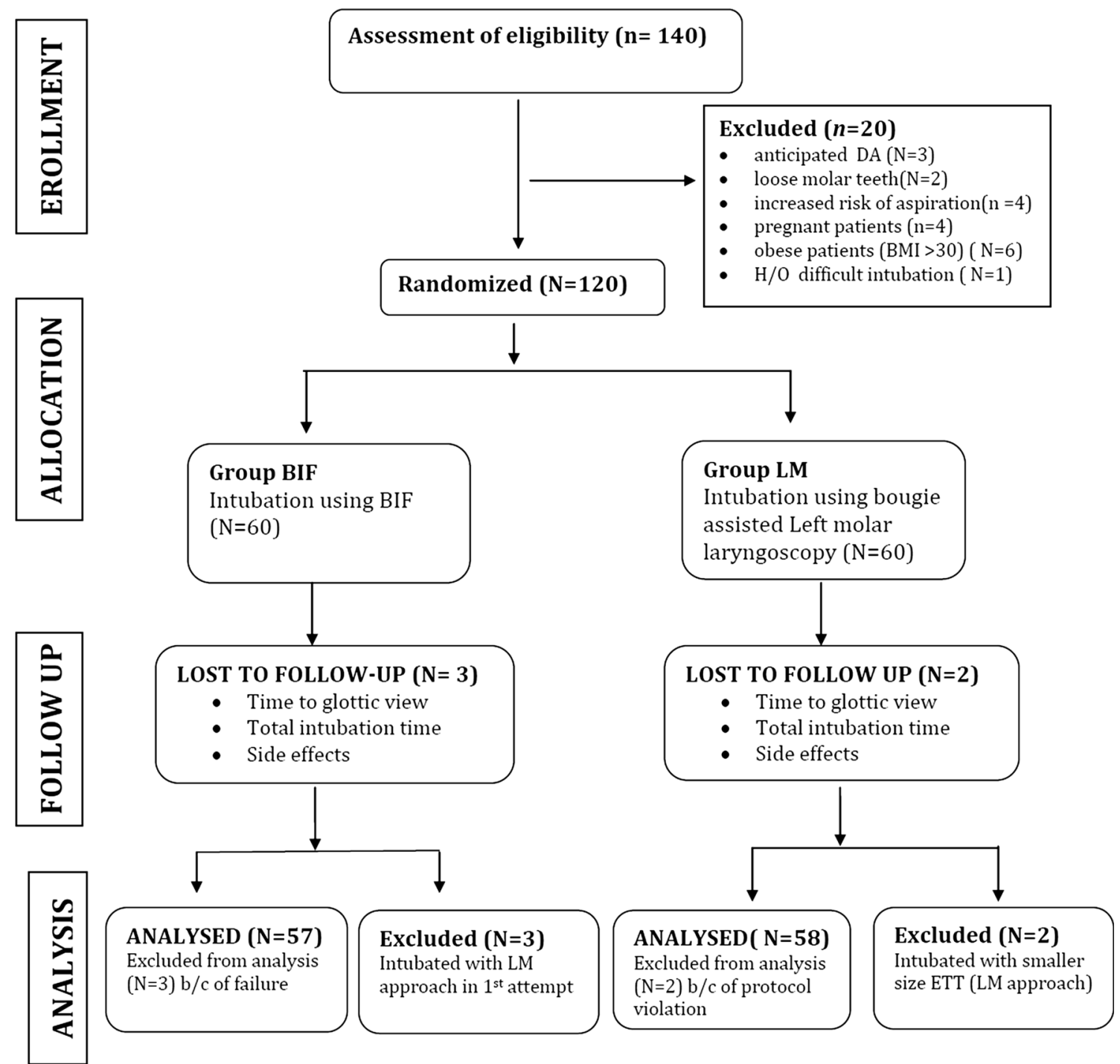

Fig. 1 CONSORT participant flow diagram. DA = difficult airway; BMI = body mass index; Group LM = left molar; Group BIF = Bonfils intubation fibrescope

lens fogging, and difficult disengagement of the ETT from the BIF. 4,21,23 We administered glycopyrrolate in all patients to deal with secretions; antifogging solution was used, and maximum jaw lift was applied to improve the oropharyngeal space for better glottic visualization. Also, an assistant disengaged the ETT from its holder and advanced the ETT further while the operator focused on the glottic view to prevent inadvertent esophageal intubation.

In our study, the operator could not advance the BIF beneath the overhanging epiglottis in two patients because a severely restricted mouth opening precluded application of a jaw lift to move the epiglottis clear of the pharynx. In one patient, the operator was distracted when the ETT connector became stuck in the slide-cone locking collar, and this resulted in an esophageal intubation. Such an occurrence has been reported previously, and a disengaging push button has been suggested as a countermeasure. ${ }^{21}$ The effectiveness of the LM approach (assisted by a tube introducer) is suggested, being as successful intubation could have been achieved in the above three cases with this simple modification of a routine technique.

Yamamoto et al. compared midline and left and right molar approaches with direct laryngoscopy and found that the LM approach with OELM reduced the incidence of difficult midline laryngoscopy from 20 to seven cases, whereas the right molar approach improved the view in only two cases. ${ }^{11}$ Cuvas et al. showed that the LM approach improved the glottic view, but $81.5 \%$ of cases could not be intubated on the first attempt. ${ }^{24}$ Bozdogan et al. also found that the LM approach improved the view in $90 \%(18 / 20)$ of patients who had a difficult midline laryngoscopy, although tracheal intubation could not be 

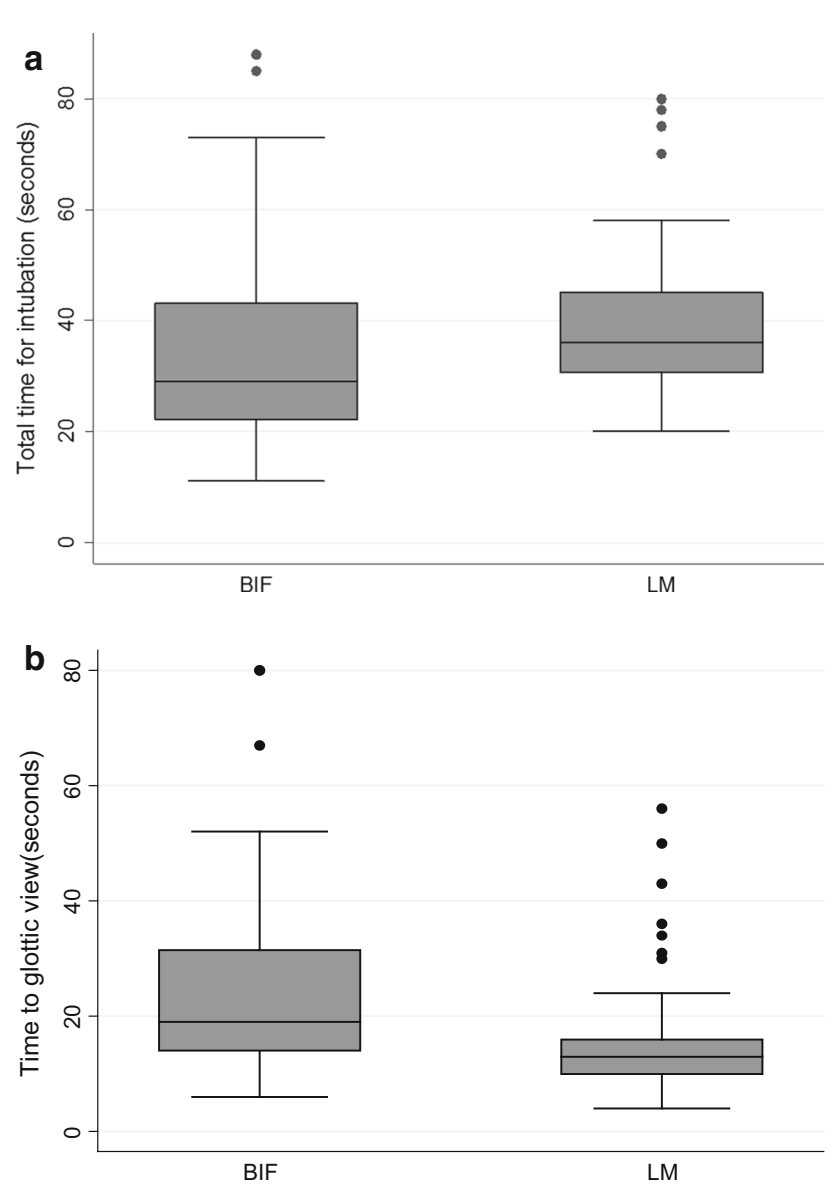

Fig. 2 a. Total intubation time. b. Time to glottic view. $\mathrm{BIF}=$ Bonfils intubation fibrescope $; \mathrm{LM}=$ left molar

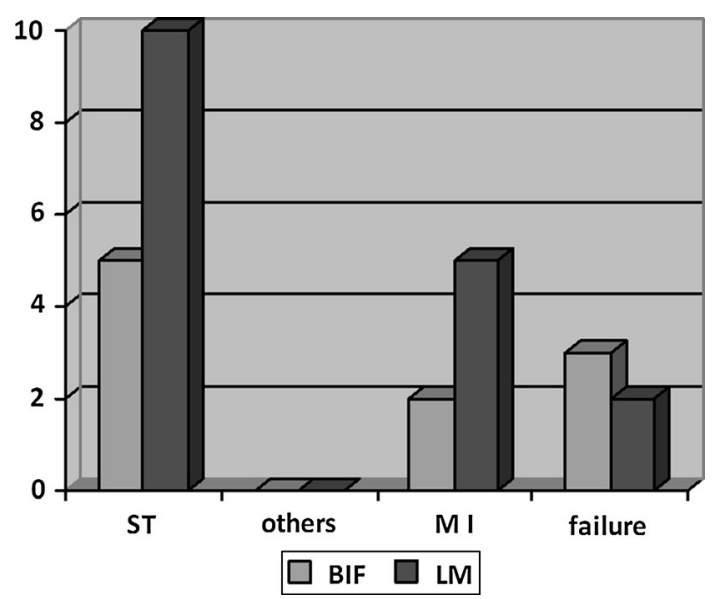

Fig. 3 Side effects. ST = sore throat; others = dental injury, esophageal intubation; $\mathrm{MI}=$ mucosal injury

performed in $35 \%(7 / 20)$ of these patients. ${ }^{13}$ The narrow space and a bulging tongue over the blade obscured the view of the glottis and made tracheal intubation difficult under vision.
Saini et al. used a stylet to improve the rate of successful intubation using LM laryngoscopy in patients with simulated limitation of cervical spine movement, but tracheal intubation failed in one patient with a CormackLehane grade 2 view. ${ }^{12}$ In our study, we achieved the same success rate (96.6\%) and similar mean (SD) time to glottic view as their study [14 (7) sec vs 15.4 (10.3) sec] despite using a rigid collar $v s$ their semi-rigid collar. ${ }^{11}$ Nevertheless, the mean (SD) total time for intubation was longer in our study than in theirs [40.4 (14) sec vs 33 (7) $\mathrm{sec}$, respectively] because of the extra time required to insert the tube introducer.

We presume that the favourable results with the LM approach in our study were because of the use of a tube introducer (stiff catheter with high-gloss low-friction outer surface and a soft atraumatic angled tip) that countered the only disadvantage associated with the LM approach, i.e., a narrow space to negotiate the tracheal tube. In two Group LM patients, the size 7 ETT could not be threaded over the tube introducer; however, an ETT with a $6.5 \mathrm{~mm}$ internal diameter could be guided easily over the tube introducer. Since protocol was violated (use of smaller size ETT), the tracheal intubations for these patients were considered failures and excluded from further analysis.

In two patients with missing left molar teeth in Group LM, the flange got stuck in the interdental cleft and precluded visualizing insertion of the tube introducer from the left side. As a result, the tube introducer was inserted from the opposite corner of the patient's mouth towards the glottis while maintaining the view from the left molar approach.

The BIF is an optical stylet with a narrow shaft that permits targeted intubation with minimal trauma. ${ }^{4,21}$ In our study, there was also less airway morbidity in Group BIF (Fig. 3), which is similar to previous reports. ${ }^{21,25}$ In Group LM, pressure may have been exerted to negotiate the tip of the laryngoscope blade between the molars, and this may have increased airway-related adverse events in this group. Nevertheless, the mucosal bleeding was minimal and selflimiting in all the cases.

Similarly, there was a lower incidence of sore throat in Group BIF than in Group LM (8.7\% vs $17.2 \%$, respectively; $P=0.25$ ) (Fig. 3). The use of the BIF approach has been found to be associated with a decreased incidence of sore throat. ${ }^{3,26}$ This may be because of reduced contact, fewer disrupting forces on the airway structures, and visualized intubation of the ETT. ${ }^{9}$ All cases of sore throat had resolved within $24 \mathrm{hr}$ in both groups.

Our study has some limitations. First, though the use of a rigid collar resulted in extremely difficult intubating conditions, the results may not be equally applicable to other difficult airway scenarios. Second, since a CormackLehane grade 4 view was not observed in our study, the 
study devices could not be evaluated under these conditions. Third, an experienced anesthesiologist performed all intubations in our study and similar success rates may not be achieved by novices.

Fourth, the difficult airway scenario in our study (decreased mouth opening and restricted neck movements) was simulated with the help of a rigid cervical collar, and all tracheal intubations were performed in patients with the rigid collar in situ. Our purpose was only to evaluate the study devices under a difficult airway situation (due to restricted mouth opening and limited neck movements) by navigating the devices around a cervical collar. But the standard practice of tracheal intubation in unstable cervical spine patients is to remove the anterior portion of the collar and intubate using manual in-line stabilization (MILS). ${ }^{27} \mathrm{We}$ do not suggest changing our normal practice of applying MILS and the use of said approaches in such patients. For this reason, we did not perform this study in patients with an unstable cervical spine, and further studies are required before we can arrive at such a conclusion. Moreover, the use of study devices may vary in different countries because of varying availability, attitudes, beliefs, practices, and economic constraints.

\section{Clinical implications}

In our simulated model of a difficult airway, we found that intubation using LM laryngoscopy with a tracheal tube introducer (readily available equipment) and OELM achieved a comparable success rate and took only slightly longer to complete than intubation using a Bonfils intubation fibrescope. Based on our experience with the LM approach, we advocate wider use of this technique.

In conclusion, both techniques described in our study were effective in managing the simulated difficult airway scenario with no serious airway complications. The total intubation time in Group LM was found to be non-inferior to that in Group BIF. We propose that the combination of left molar laryngoscopy, along with OELM and a tube introducer, can be a reliable cost-effective option for intubation in difficult airway scenarios similar to our setting.

Conflicts of interest None declared.

\section{References}

1. Hastings RH, Wood PR. Head extension and laryngeal view during laryngoscopy with cervical spine stabilization maneuvers. Anesthesiology 1984; 80: 825-31.

2. Goutcher CM, Lochhead V. Reduction in mouth opening with semi-rigid collars. Br J Anaesth 2005; 95: 344-8.
3. Bein B, Worthmann F, Scholz J, et al. A comparison of the intubating laryngeal mask airway and the Bonfils intubation fibrescope in patients with predicted difficult airways. Anaesthesia 2004; 59: 668-74.

4. Byhahn C, Nemetz S, Breitkreutz, R, Zwissler B, Kaufmann M, Meininger D. Brief report: tracheal intubation using the Bonfils intubation fibrescope or direct laryngoscopy for patients with a simulated difficult airway. Can J Anesth 2008; 55: 232-7.

5. Bein B, Yan M, Tonner PH, Scholz J, Steinfath M, Dorges V. Tracheal intubation using the Bonfils intubation fibrescope after failed direct laryngoscopy. Anaesthesia 2004; 59: 1207-9.

6. Wahlen BM, Gercek E. Three dimensional cervical spine movement during intubation using the Macintosh and Bullard $^{\mathrm{TM}}$ laryngoscopes, the Bonfils fibrescope and the intubating laryngeal mask airway. Eur J Anaesthesiol 2004; 21 : 907-13.

7. Rudolph C, Schneider JP, Wallenborn J, Schaffranietz L. Movement of the upper cervical spine during laryngoscopy: a comparison of the Bonfils intubation fibrescope and the Macintosh laryngoscope. Anaesthesia 2005; 60: 668-72.

8. Piepho T, Noppens RR, Heid F, Werner C, Thierbach AR. Rigid fibrescope Bonfils: use in simulated difficult airway by novices. Scand J Trauma Resusc Emerg Med 2009; 17: 33.

9. Thong SY, Wong TG. Clinical uses of the Bonfils Retromolar Intubation Fiberscope: a review. Anesth Analg 2012; 115: 855-66.

10. Law JA, Broemling N, Cooper RM, et al.; Canadian Airway Focus Group. The difficult airway with recommendations for management - part 1 - difficult tracheal intubation encountered in an unconscious/induced patient. Can J Anesth 2013; 60: 1089118.

11. Yamamoto K, Tsubokawa T, Ohmura S, Itoh H, Kobayashi T. Left-molar approach improves the laryngeal view in patients with difficult laryngoscopy. Anesthesiology 2000; 92: 70-4.

12. Saini $S$, Bala $R$, Singh $R$. Left-molar approach improves laryngeal view in patients with simulated limitation of cervical movements. Acta Anaesthesiol Scand 2008; 52: 829-33.

13. Bozdogan N, Sener M, Bilen A, Turkoz A, Donmez A, Arslan G. Does left molar approach to laryngoscopy make difficult intubation easier than conventional midline approach? Eur J Anaesthesiol 2008; 25: 681-4.

14. Samsoon GL, Young JR. Difficult tracheal intubation: a retrospective study. Anaesthesia 1987; 42: 487-90.

15. Corbanese $U$, Morossi $M$. The Bonfils intubation fibrescope: clinical evaluation and consideration of the learning curve. Eur $\mathbf{J}$ Anaesthesiol 2009; 26: 622-4.

16. Cormack RS, Lehane J. Difficult tracheal intubation in obstetrics. Anaesthesia 1984; 39: 1105-11.

17. Benumof JL, Cooper SD. Quantitative improvement in laryngoscopic view by optimal external laryngeal manipulation. J Clin Anesth 1996; 8: 136-40.

18. Sumathi PA, Shenoy T, Ambareesha M, Krishna HM. Controlled comparison between betamethasone gel and lidocaine jelly applied over tracheal tube to reduce postoperative sore throat, cough, and hoarseness of voice. Br J Anaesth 2008; 100: 215-8.

19. Wakeling $H G$, Nightingale $J$. The intubating laryngeal mask airway does not facilitate tracheal intubation in the presence of a neck collar in simulated trauma. Br J Anaesth 2000; 84: 254-6.

20. Heath KJ. The effect of laryngoscopy of different cervical spine immobilisation techniques. Anaesthesia 1994; 49: 843-5.

21. Halligan $M$, Charters $P$. A clinical evaluation of the Bonfils intubation fibrescope. Anaesthesia 2003; 58: 1087-91.

22. Mihai R, Blair E, Kay H, Cook TM. A quantitative review and meta-analysis of performance of non-standard laryngoscopes and rigid fibreoptic intubation aids. Anaesthesia 2008; 63: 745-60. 
23. Wong TG. The Bonfils retromolar intubation fiberscope: advantages and practical aspects of its use. AJA- Online.com 2011; 12: 40-7.

24. Cuvas O, Basar H, Gursoy N, Culhaoglu S, Demir A. Left-molar approach for direct laryngoscopy: is it easy? J Anesth 2009; 23: 36-40.

25. Rudolph C, Henn-Beilharz A, Gottschall $R$, Wallenborn $J$, Schaffranietz, $L$. The unanticipated difficult intubation: rigid or flexible endoscope? Minerva Anesthesiol 2007; 73: 567-74.
26. Webb A, Kolawole H, Leong S, Loughnan TE, Crofts T, Bowden $C$. Comparison of Bonfils and Levistan optical stylets for tracheal intubation: a clinical study. Anesth Intensive Care 2011; 39: 1093-7.

27. Manoach $S$, Paladino $L$. Manual in line stabilization for acute airway management of suspected cervical injury: historical review and current questions. Ann Emerg Med 2007; 50: $236-45$. 